\author{
Rev. Giulio Maspero* \\ Pontificia Università della Santa Croce, Roma
}

\title{
I PADRI DELLA CHIESA E LA METAFISICA: UNA RELAZIONE INELUDIBILE
}

\begin{abstract}
Sono molto importanti le riflessioni sull'uso della metafisica nell'insegnamento dei Padri della Chiesa, perché questo legame è molto radicato nell'esegesi fatta da loro. Questa è stata contestata soprattutto in ambito della Riforma che sosteneva che il linguaggio biblico fosse una derivazione della filosofia greca pagana. Padri della Chiesa non solo hanno lavorato sulla metafisica stessa, ma hanno anche modificato la sua forma classica, elaborandola in un certo modo come una nuova ontologia. Questo ha aiutato molto nella descrizione teologica di Dio Trino e Uno in modo molto più preciso. Su questa base hanno interpretato molto più in profondità tante questioni teologiche, facendole vedere in una nuova luce, ad es. l'opera della creazione, il mistero dell'uomo e la mariologia.
\end{abstract}

\section{INTRODUZIONE}

Parlare di Padri della Chiesa e Metafisica non è banale perché tale rapporto è profondamente radicato nella loro esegesi che è stata seriamente criticata, particolarmente in ambito riformato, in base al principio che il linguaggio biblico sarebbe stato soppiantato da quello della filosofia pagana greca. ${ }^{1}$ Ma la posizione di rifiuto dialettico nei confronti della componente metafisica del pensiero cristiano prendeva le mosse da un approccio alla filosofia tecnicistico, segnato dalla scolastica e dal nominalismo.

Eppure, se si ammette che la dottrina trinitaria non è altro che una riformulazione della fede rivelata attraverso il Vangelo e la vita della Chiesa, non si può elu-

* Rev. Giulio Maspero - sacerdote cattolico, professore ordinario di Teologia Dogmatica, Pontificia Università della Santa Croce; e-mail: maspero@pusc.it; ORCID: 0000-0001-6827-4436.

1 Si vedano, ad esempio, le influenti critiche di Adolf von Harnack, che hanno segnato in modo determinante gli studi successivi ed esprimono in un ceto senso lo spirito di un'epoca: A. von Harnack, Lehrbuch der Dogmengeschichte I, Freiburg i. B. 1888, pp. ix-x. 
dere la questione del rapporto tra i Padri e la Metafisica. ${ }^{2}$ La Trinità, infatti, deve prendere il posto di quel primo principio ricercato dai filosofi. Evidentemente la dottrina cristiana supera infinitamente ogni risultato precedente, ma laicamente dobbiamo assicurare che la questione trinitaria non si ponga su un piano assolutamente insignificante per l'uomo non credente. Altrimenti, come sarebbe possibile essere Chiesa in uscita, capace di rivolgersi a chi non ha incontrato ancora il Signore, Verbo di Dio che si è fatto carne? ${ }^{3}$

Ancora di più, se le cose stanno così, non ci si può fermare ad una riformulazione della metafisica che rappresenti un'ontologia della Trinità, ma bisogna sviluppare anche un'ontologia dalla Trinità, cioè una radicale considerazione dell'essere del mondo creato alla luce che promana dalla rivelazione trinitaria. ${ }^{4}$ La proposta qui avanzata è che la chiave di questa nuova metafisica sviluppata dai Padri della Chiesa per annunciare il Vangelo a chi non crede e mostrare la significatività del dogma cristiano per la vita dell'uomo è la relazione. Si tratta in concreto di un'ontologia relazionale sviluppata dai Padri della Chiesta durante le dispute che hanno condotto alla formulazione del dogma trinitario con i Concili di Nicea e Costantinopoli nel sec. IV. ${ }^{5}$

\section{METAFISICA E SCRITTURA}

La prospettiva dalla quale i Padri della Chiesa parlano di metafisica è quella che loro indicavano con l'espressione "le nozioni comuni". Si tratta dell'insieme di conoscenze di base condivise dall'uomo nel suo vivere comune. La metafisica, infatti, si usa costantemente, nell'esistenza quotidiana. Si pensi, ad esempio, al ristorante, quando si ordina carne piuttosto che pesce. Se il cameriere sbaglia a portare il piatto, le proteste sono sempre fondate su un giudizio metafisico, per quanto elementare. E lo stesso si può dire della teologia: anche se un essere umano non la studia a livello scientifico, le scelte concrete che ciascuno compie nella propria esistenza implicano una posizione teologica. Se si investe tutta la propria vita per cercare di guadagnare denaro, facendo di esso il senso della propria esistenza, allora il denaro stesso sarà di fatto considerato come il proprio dio, un idolo in questo caso, anche se non si è consci di tale opzione teologica.

Sul nuovo clima sorto nel secolo scorso a proposito del rapporto tra metafisica e patristica, si veda Ph.G. Renczes, La patristica e la metafisica nel secolo XX, Gregorianum 90 (2009), 76-85. Cfr. Papa Francesco, Evangelii gaudium, nn. 20-24.

Cfr. P. Coda, L'ontologia trinitaria: cos'è?, Sophia 4 (2012), 159-179.

Per un'analisi storica della questione, si veda G. Maspero, Essere e relazione, Roma 2013. 
Da tale prospettiva, la Bibbia, e il Vangelo in particolare, si giocano tutti su una questione metafisica. Dal momento in cui Adamo è chiamato a dare il nome ai diversi animali creati da Dio senza trovare qualcuno a cui dare del $t u$, fino all'incontro di Mosé al roveto ardente e alla manna nel deserto, tutto l'Antico Testamento parla di una questione metafisica. Non a caso la domanda fondamentale di tale scienza è "cosa è?", significato proprio di man hu (Ex 16:15). Israele deve cogliere praticamente che Dio è una realtà totalmente altra rispetto al mondo, cioè che è unico e assolutamente trascendente, a differenza delle divinità pagane, che erano personificazioni di forze naturali.

Nel Nuovo Testamento il problema è ancora più acuto, perché Gesù dice di essere proprio Dio, l'unico Dio. In tutte le lingue indo-europee esistono due radici fondamentali per riferirsi al padre: pater e atta. ${ }^{6}$ In inglese si tratta dei termini father e $d a d$. Il primo implica solo origine e si può quindi predicare di realtà metafisiche differenti: Adamo poteva chiamare così il Creatore, gli ebrei potevano dire che Dio era il Padre del popolo, così come oggi si può dire che il fondatore di un'azienda è il padre di essa o che un politico è il padre della patria. Il secondo termine, più famigliare, implica invece non solo origine, ma anche consaguineità. Pater poteva essere riferito al capo-tribù, agli antenati, e, in primo luogo, agli dèi. L'atta, invece, è solo il proprio papà. Ciò rivela che quanto Gesù chiama abbà l'unico Dio Creatore del cielo e della terra sta ponendo un serissimo problema metafisico, il più serio ad essere mai stato posto. La croce stessa è conseguenza di questo scandalo metafisico, dettato dal fatto che Gesù, il quale è perfettamente uomo, dice di essere Dio, cioè un'altra cosa. La risurrezione sarà, così, la prova della necessità di modificare la metafisica classica, accostando alla domanda sul che cosa, quella sul chi. Gesù, infatti, è la stessa cosa del Padre, ma è un chi diverso, un'altra persona. Il Mistero Pasquale può essere considerato, da tale prospettiva, il più grande e fondamentale giudizio metafisico della storia. La Scrittura stessa pone, dunque, i suoi lettori di fronte ad una sfida filosofica, perché il Dio uno e trino è un'unica sostanza, infinita ed eterna, ma in tre Persone: tre chi differenti che sono una sola cosa.

E tale ricomprensione della metafisica nasce dalla Scrittura stessa. Piero Coda ha acutamente osservato, infatti, che le dispute trinitarie del sec. IV possono essere lette come discussione riguardo all' esegesi del prologo giovanneo. ${ }^{7}$ In effetti quanto visto fino ad ora trova la sua radice proprio nell'incipit del quarto vangelo, collocandosi, dunque, a un livello fondante, quindi, in qualche modo anche ineludibile per il pensiero cristiano.

\footnotetext{
6 Cfr. E. Benveniste, Le vocabulaire des institutions indo-européennes, I, Paris 1969, pp. 210-211.

7 Cfr. P. Coda, Per una lettura trinitaria del prologo di Giovanni, Milano 2007, pp. 85-94.
} 
Se si legge l'insieme del prologo giovanneo si può notare la sua struttura chiastica. Come nella figura della lettera greca chi, si osserva che il testo può essere diviso in due grandi parti che si incontrano nell'affermazione in Gv 1,11-13 che chi accoglie il Logos che si fa carne diventa figlio di Dio. Nella prima metà il protagonista è il Logos che (a) è nell'arché (Gv 1,1-2), (b) è responsabile della creazione che però viene turbata dal peccato $(\mathrm{Gv} 1,3-5)$, (c) è annunciato dal Battista che rende testimonianza alla luce $(\mathrm{Gv} 1,6-8)$ e (d) alla fine viene nel mondo come luce vera (Gv 1,9-10). Il movimento è qui dall'alto verso il basso, dall'immanenza all'economia. Nella seconda metà, invece, il percorso di ripete all'inverso, traducendo le stesse tappe della prima parte in termini storici e concreti, per svelare alla fine l'identità del Logos. Così (d') il Logos non solo viene nel mondo come luce, ma si fa carne (Gv 1,14), (c') e il Battista rende testimonianza proprio a questa identità $(\mathrm{Gv} 1,15)$, in modo tale che (b') il senso della creazione può essere letto pienamente nella nuova creazione che nel dono della grazia sostituisce la legge mosaica (Gv 1,16-17), per concludere con la rivelazione che l'arché è il seno del Padre e il Logos è l'Unigenito (Gv 1,18). Nella lettura le dieci parole della Legge sono poste in parallelo alle dieci parole con le quali Dio crea il mondo all'inizio della Genesi. E tale operazione sembra fedele all'intenzione dell'autore che omette l'articolo nell'espressione en arché, come grammaticalmente sarebbe stato meglio in greco, per richiamare il be rescit di Gn 1,1. Inoltre il Logos stesso richiama il dabar con il quale Dio ha creato ogni $\operatorname{cosa} .^{8}$

I Padri hanno preso metafisicamente sul serio il testo giovanneo e lo hanno posto a fondamento di una nuova ontologia, nella quale l'arché ricercato dai filosofi rivela in Cristo di avere un'immanenza abitata dal Logos stesso. Questi, allora, è nel primo principio in quanto è rivolto a Lui (pros ton). Quest'ultima espressione greca può essere letta come riformulazione dell'espressione che da Aristotele in poi indica la relazione, cioè il pros $t i{ }^{9}{ }^{9}$ 'essere rivolto a qualcosa, che secondo Giovanni è però quell'essere rivolto verso Qualcuno, in concreto verso il Padre, che caratterizza e costituisce il Figlio. La seconda Persona della Trinità è, infatti, pura relazione perché è Figlio eterno del Padre stesso e da sempre abita il Suo seno. Quindi sia l'arché sia il logos della metafisica devono essere riletti alla luce di una concezione della relazione che non è più mero accidente ma sussiste nell'unica infinita ed assoluta sostanza divina.

Tale ricomprensione relazionale implica che solo l'Unigenito può rivelare Dio, farne l'esegesi (cfr. Gv 1,18). Da questa prospettiva la posizione teologica dei Padri è caratterizzata da un vero e proprio gap ontologico che sarà illustrato

Cfr. G. Maspero, Uno perché trino, Siena 2011, pp. 33-35.

9 Cfr. Aristotele, Categoriae, 1b 26-2a1 e 6a 36-37. 
nella sezione successiva e dall'apofatismo, che del gap è conseguenza gnoseologica. Tutto questo può essere ricondotto proprio al Prologo e, pertanto, essere considerato definitorio dell'epistemologia cristiana. All'Essere stesso, nella sua profondità, si può accedere solo attraverso la relazione personale con il Figlio incarnato, perché l'essere stesso è relazione. L'apofatismo non appare più come negazione, ma come affermazione positiva dell'inesauribile profondità ontologica di Dio. L'approccio teologico non può, così, essere considerato contraddittorio rispetto a quello filosofico, perché i Padri vedono l'essere da dentro, attraverso una relazione concreta senza la quale non potrebbero aver accesso a tale profondità. L'apofatismo non nega il valore della ragione, ma solo segnala che il suo limite può anche essere una soglia, cioè che là dove il pensiero metafisico entra in crisi si spalancano come finestre nuove possibilità. La ratio, infatti, si compie così in relatio.

Si noti che il Prologo può essere letto come riferimento contemporaneo sia agli elementi biblici del be rescit e del dabar, sia alle categorie metafisiche dell'arché e del logos, e forse persino alla riconfigurazione ontologica del pros ti come pros ton personale. ${ }^{10}$

\section{L'ONTOLOGIA DEL GAP}

Dunque, se la questione metafisica non è una sovrastruttura rispetto alla Scrittura, ma è ad essa intrinseca, è naturale che le dispute trinitarie dei primi quattro secoli siano caratterizzate da un forte sviluppo del pensiero ontologico. Con questo termine non ci si riferisce a Heiddegger e all'accezione introdotta dalla sua ermeneutica, piuttosto con esso si indica secondo etimologia "il logos sull'essere" per distinguerlo dalla metafisica greca stessa. Questa può essere considerata un caso particolare di ontologia essenzialmente caratterizzata come ricerca della realtà che fonda causalmente ta physika, cioè la natura. Platone ed Aristotele, infatti, inaugurano una seconda navigazione, che si stacca dai primi risultati filosofici dei presocratici, perché spinge la ricerca del primo principio al di là del cosmo visibile stesso, per rinvenire l'arché nell'ambito della dimensione intellegibile. Le idee platoniche o la forma aristotelica sono sempre principi metafisici che fondano il mondo visibile nell'invisibile. Platone dice che gli uomini sono alberi con

10 In questo senso il metodo dei Padri della Chiesa, i quali usano gli elementi di verità presenti nella cultura pagana, così come ha fatto anche l'arte cristiana, è contenuto in nuce già nel Prologo di Giovanni. Il metodo patristico è stato studiato magistralmente in Ch. Gnilka, Chrêsis: die Methode der Kirchenväter im Umgang mit der antiken Kultur: Der Begriff des "rechten Gebrauchs", Basel 2012. 
le radici in alto, riconducendo lo sguardo metafisico alla memoria. ${ }^{11}$ Aristotele evidenzia, invece, l'atto, sviluppando l'intuizione platonica nella sequenza di motori che risale la scala metafisica verso la purezza attuale assoluta costituita dal Motore Immobile, pensiero di pensiero. ${ }^{12}$

Così, sia Platone sia Aristotele si sono protesi con il pensiero fino all'arché procedendo di causa in causa. Il termine greco che indica queste ultime è proprio logos. E la possibilità di tale ascesa va individuata in una struttura comune al pensiero di entrambi i grandi pensatori, secondo i quali il primo principio e il mondo risultano essere collegati da una serie di nessi causali necessari, indicati dal termine logos appunto, in modo tale che dio e il cosmo costituiscono un unico livello metafisico, articolato in una scala di densità ontologica discendente a partire dalla perfezione dell'arché stesso. In tale quadro filosofico, il principio di individuazione è sempre legato ad un elemento di imperfezione, poiché solo l'universale può essere identificato con la perfetta intelligibilità. Così in Platone è la materia, plasmata dal demiurgo a partire dall'archetipo ideale, ad introdurre il determinato, mentre in Aristotele ciò si gioca nell'interazione della coppia potenza-atto. Si tenga presente che quest'ontologia che abbraccia in unico livello sia il primo principio sia il mondo è caratterizzata da finitezza ed eternità.

Dalla prospettiva biblica questi due elementi metafisici dovevano essere radicalmente superati, perché il Creatore del mondo è infinito ed eterno, mentre il mondo è finito e temporale. Ciò spinse ad un lungo processo, iniziato da Filone e portato avanti dai Padri anche in interazione con il neopitagorismo, lo stoicismo e il medio- e il neo-platonismo, per passare ad una concezione ontologica a due livelli. Tra Dio e il mondo era necessario, infatti, introdurre un autentico gap metafisico, uno iato infinito che distinguesse l'unica ontologia caratterizzata da infinitezza ed eternità rispetto all'ontologia del mondo creato, segnato radicalmente da un inizio nel tempo e dalla finitudine.

Proprio questo passaggio è fondamentale nella posizione del problema dell'ontologia trinitaria. Infatti, l'introduzione di due diverse ontologie per il primo principio e per il mondo rende inevitabile la discussione del rapporto tra le due. Il punto è che in un certo senso tale rapporto è già espressione della concezione dell'arché: se quest'ultimo è inteso a partire dalla necessità, è perfettamente coerente che il mondo sia ad esso necessariamente connesso e che ne sia distinto solo attraverso una degenerazione. L'unica forma di identità possibile è, infatti, quella ideale e la sua negazione dialettica. Se l'essere è sic et simpliciter identificato con l'intelligibile, allora tertium non datur. Ma se l'essere è eccedente rispetto alla

11 Cfr. Platone, Timaeus, 90 a-b.

12 Cfr. Aristotele, Metaphysica, XII, 7: 1072a, 21-26. 
conoscenza dell'uomo, allora si ha una terza possibilità che è proprio quella della relazione, in base alla quale due realtà possono essere distinte per la stessa ragione che le unisce. Il gap, infatti, implica che il rapporto tra il primo principio e il mondo debba essere intesto in senso relazionale, senza introdurre un'opposizione tra creatura e Creatore, quindi senza svalutare la prima per affermare il secondo.

Quanto sia radicale la novità ontologica è mostrato proprio dalla pretesa di Gesù, della cui veridicità è prova la risurrezione, di essere una cosa sola con il Padre, essendo un chi diverso da Lui. La "Dad-hood" richiama, infatti, una forma nuova di identità, che non era mai stata conosciuta prima, poiché il Figlio non può essere detto "dio" in quanto appartenente alla stessa categoria, come avviene a livello categoriale per una specie. Nel caso del primo principio assoluto, per definizione, questa conta un solo elemento. La tag "dio" non può essere attribuita a più membri secondo una identità di tipo platonico, per la quale un elemento è identificato dalla sua appartenenza alla categoria che sottostà ad un'idea, ad esempio un determinato cavallo che è tale perché corrisponde all'idea di "cavallinità". Né nel caso di Dio si può applicare l'identità dialettica, in quanto il gap impedisce di definire ciò che è Dio per contrasto rispetto ad altro. Il mondo, infatti, non è più necessariamente connesso al primo principio e non è da sempre.

Invece, Gesù di Nazareth afferma "prima che Abramo fosse, io sono" (Gv 8,58), cioè dice di essere Figlio eterno del Padre eterno, il quale non è Dio se non in Lui. Dice "Io sono nel Padre e il Padre è in me" (Gv 14,10) ponendo una sfida immensa alla metafisica classica per la quale l'accidente e la partecipazione erano legate proprio all'inerire ad una sostanza altra, cioè all'essere in alio. Ma qui il Padre stesso è in Filio, ragion per cui si impone la conclusione che il Figlio è eterno, cioè è l'assoluto, come poi si ripeterà per lo Spirito Santo, che in quanto Spirito del Padre e del Figlio è in loro, in quanto la prima e la seconda Persona sono in Lui.

Questa nuova forma di identità richiederà lo sviluppo di una nuova ontologia, che chiamiamo trinitaria proprio perché affronta il problema del rapporto tra il primo principio e il mondo alla luce del gap introdotto dai Padri della Chiesa nello sviluppo del dogma trinitario. A poco a poco essi giunsero ad una comprensione relazionale dell'identità delle tre Persone divine a partire dal dato scritturistico dei loro nomi propri rivelati, i quali dicono relazione. Padre, Figlio e Spirito-Amore fanno, infatti, riferimento l'uno all'altro.

Ma ciò ha richiesto un percorso che sinteticamente si può far iniziare da Giustino, il quale come buon filosofo è ben conscio della sfida. La sua stessa biografia lo porta a spiegare la distinzione tra il Padre e il Figlio in termini di logos, riprendendo il prologo giovanneo. Il Padre da sempre pensa il modo in modo tale che tale pensiero è suo, è in Lui, ma è distinto da Lui. Così, ogni verità conosciuta 
dall'uomo prima dell'incarnazione può essere riletta come espressione del logos e da essere riconosciuto, quindi, patrimonio cristiano. ${ }^{13}$ La soluzione presentava due lati oscuri principali: a) l'esistenza del Figlio, per quanto eterna, veniva legata necessariamente al cosmo, a causa dell'identificazione con il Logos; b) la piena divinità rimaneva esclusiva solo del Padre, il quale per questo non era detto Persona, in quanto il termine indicava limitazione e veniva predicato solo del Figlio. ${ }^{14}$ Per Giustino si può dire non che è il mondo ad essere per il Logos, ma esattamente il contrario. La distinzione tra le Persone divine è ancora platonicamente veicolo di subordinazione. È estremamente significativo come la distinzione intratrinitaria e quella tra il primo principio e il mondo appaiano qui sovrapposte e non perfettamente distinte. Il gap non è, infatti, ancora stato formulato in tutta la sua forza.

Per questo si rivelerà essenziale il contributo di Origene, il quale supera la teologia del logos degli apologisti ${ }^{15}$ per formulare la chiara appartenenza ad un unico livello ontologico del Padre, del Figlio e dello Spirito Santo. ${ }^{16}$ Afferma, infatti, che nella Trinità non si dà il più e il meno, formula metafisicamente riservata alla pienezza sostanziale. ${ }^{17}$ Il gap è qui perfettamente espresso. Eppure proprio nel contesto della teologia trinitaria di Origene apparve con forza la necessità di un ulteriore sviluppo metafisico che portasse ad una vera e propria ontologia trinitaria. Infatti, l'Alessandrino cerca di distinguere le tre Persone divine dal creato attribuendo solo a loro la pura spiritualità. ${ }^{18}$ Sia gli angeli sia gli uomini, invece, sarebbero caratterizzati da un elemento corporale, tanto più sottile quanto più loro fossero prossimi alla perfezione. Come si nota immediatamente, mancava una concezione della physis forte come quella che sarà sviluppata da Atanasio nel IV secolo. Così, secondo Origene, il Figlio è Dio di secondo grado rispetto al Padre e né Lui né lo Spirito si identificano con gli attributi divini del Padre, ma solo ne

13 Cfr. Giustino, Apologia, I, 46, 2, 1-3, 6.

14 Cfr. id., Dialogus cum Tryphone, 127.

15 Cfr. D. Pazzini, In principio era il Logos: Origene e il prologo del Vangelo di Giovanni, Brescia 1983; id., L'interpretazione del Prologo di Giovanni in Origene e nella patristica greca, Annali di storia dell'esegesi 11 (1994), 45-56.

16 Per una critica ad ogni possibile lettura subordinazionista di Origene, si veda I. Ramelli, Origen's anti-Subordinationism and its Heritage in the nicene and Cappadocian Line, Vigiliae christianae 65 (2011), 21-49.

17 Cfr. M. Simonetti, voce "Spirito Santo", in: Dizionario Origene, ed. A. Monaci Castagno, Roma 2000, pp. 450-456.

18 Per Origene è fondamentale l'esegesi di Gv 4,24 in funzione antistoica. Si veda Origene, Commentarii in evangelium Joannis, XIII, 21, 140, 1-12. Per gli stoici, infatti, lo pneuma era corporale, in modo tale da spingere l'Alessandrino a un'affermazione netta dell'autentica spiritualità di Dio e, quindi, delle tre Persone divine, che in questo si differenziano assolutamente sia dagli uomini sia dagli angeli, creature tutte caratterizzate da una dimensione corporale, per quanto sottile. 
partecipano. ${ }^{19}$ Si può dire che l'Alessandrino avverte con forza la necessità di distinguere l'ontologia della Trinità da quella del creato, ma i suoi tentativi, per quanto geniali, continuano a proiettare parzialmente in Dio la metafisica sviluppata in ambito categoriale. La strutturazione del primo principio, infatti, evidenzia un'insufficienza ontologica a livello della distinzione immanente delle tre Persone divine ancora riferita ad una deficienza metafisica. Senza che si dia una vera e propria subordinazione, il Padre, il Figlio e lo Spirito appaiono ancora disposti in una scala ontologica di grado decrescente.

La crisi ariana prenderà le mosse proprio da tale tensione espressiva. Sia Ario sia Eunomio si appelleranno alle espressioni di Origene, portando la gradazione ontologica fuori dall'immanenza divina. Se il Figlio è un dio di seconda categoria, allora, in nome del gap, che la dottrina della creazione esige, Egli deve essere una creatura, e quindi originato nel tempo. Ma allora ci deve essere stato un tempo in cui la seconda Persona non era e, quindi, Dio non era Padre. Atanasio risponde con l'introduzione della teologia delle nature, in base alla quale la Trinità si identifica con l'unica natura eterna ed infinita, che un gap assoluto separa da tutte le altre nature create nel tempo e finite. In altri termini, l'Alessandrino esclude che ci possano essere zone grigie all'interno del gap stesso. La trasmissione della perfetta divinità nell'immanenza divina è, invece, assicurata da una proporzionalità perfetta per la quale il Figlio-Logos è in rapporto con il Padre proprio come lo Spirito è in rapporto con il Figlio-Logos.$^{20} \mathrm{Si}$ tratta già dell'elaborazione di una grande novità ontologica che riesce a rispondere agli argomenti ariani proprio a partire dalla funzione proporzionale del logos anche nella tradizione platonico-pitagorica e geometrica. Tuttavia tale soluzione riceverà le critiche degli pneumatomachi, cioè di quel gruppo eterodosso che accettava la divinità del Figlio, a partire dal fatto che fosse generato e, quindi, della stessa natura del Padre, ma che negava la divinità della terza Persona, proprio perché questa non è figlio. Le critiche cercavano di mettere in crisi il pensiero trinitario affermando un aut-aut: o la seconda Persona non è l'Unigenito, perché ha nello Spirito un fratello, oppure il Padre deve essere nonno dello Spirito stesso, in quanto genitore di chi lo ha generato. La dimostrazione era per assurdo, in quanto entrambe le conclusioni erano manifestamente inaccettabili. ${ }^{21}$

Entra qui in scena il pensiero cappadoce, che avrà la forza di presentare, in particolare con i due Gregorii, una vera e propria ricomprensione dell'ontologia in chiave relazionale. Il punto è proprio legato alla necessità di distinguere le Persone divine nell'immanenza solo a partire dalla differenza delle loro relazioni

19 Cfr. Origene, Commentarii in evangelium Joannis, II, 10, 76, $2-7$.

20 Cfr. Atanasio, Epistula ad Serapionem, II, 10,2.

${ }^{21}$ Cfr. ibid., III, 1,3. 
di origine. ${ }^{22}$ Ma questo richiedeva di rivedere la metafisica aristotelica su un punto capitale: per lo Stagirita la relazione non solo era un semplice accidente, ma addirittura era il minimo tra di essi, in quanto per sussistere non solo aveva bisogno di una sostanza, ma ben di due, tanto da escludere che la sostanza stessa potesse appartenere ai relativi. ${ }^{23}$ La relazione era concepita come escrescenza della sostanza, la cui perdita in nulla poteva modificare la sostanza stessa. Per i Cappadoci, invece, i nomi propri delle Persone divine dicono relazione, introducendo una distinzione all'interno dell'unica sostanza eterna ed infinita. La novità radicale di tale distinzione consisteva nel fatto che essa implicasse la perfetta identità di ciascuno dei distinti con gli attributi divini, di per sé unici. Il Padre, il Figlio e lo Spirito sono, dunque, la stessa cosa, Dio, ma l'uno non è l'altro perché le relazioni insite nei loro stessi nomi li distinguono.

La novità è formulata in termini formalmente metafisici da Gregorio di Nissa, il quale si trova a fronteggiare l'uso di schesis da parte di Eunomio proprio al fine di mostrare la presunta differenza sostanziale delle tre Persone divine. ${ }^{24}$ Così il fratello di Basilio afferma chiaramente che il Logos appartiene ai relativi (ta pros ti) e che la caratteristica che distingue ogni Persona dalle altre è il pôs einai, cioè il suo modo di sussistenza, la relazione attraverso la quale possiede eternamente e perfettamente l'essere infinito ed eterno che è Dio. ${ }^{25} \mathrm{Il}$ pôs einai stesso è una modifica del pôs echein stoico, che è portato dal livello dell'avere, cui etimologicamente si richiama anche la schesis, a quello dell'essere. Così, il fatto che il Figlio è nel Padre come il Padre è nel Figlio viene letto ora non più come prova a partire dalla metafisica aristotelica della loro differenza sostanziale, ma piuttosto come affermazione della loro consustanzialità a partire da una nuova ontologia nella quale la relazione può abitare l'immanenza della sostanza divina. Per questo il Logos prima, e lo Spirito dopo, passeranno dall'essere figure di mediazione ontologica che collegano i diversi livelli ontologici di Dio e del mondo, ad abitare e definire l'immanenza divina. Gregorio di Nissa dovrà spingersi a presentare la terza Persona come la potestà regale comunicata dal Padre al Figlio nell'atto della generazione per il quale il Figlio è Re proprio come il Padre è Re. Di più, la seconda Persona restituisce alla prima eternamente e perfettamente tale potestà che può anche chiamarsi Gloria. ${ }^{26}$ Analogamente anche Gregorio di Nazianzo presenta lo Spirito tra il Padre e il Figlio, in modo tale da superare ogni possibile critica da parte degli pneumatomachi che riconoscevano la divinità delle prime due Persone

22 Cfr. Gregorio di Nazianzo, Oratio 31 (De Spiritu Sancto), 9.

23 Cfr. Aristotele, Ethica Nicomachea, $1096^{\circ}, 21-22$.

24 Cfr. Gregorio di Nissa, Eunomio, in: id, Contra Eunomium, I, 151,1-152,1.

25 Cfr. Gregorio di Nissa, Contra Eunomium, II, 1, 386, 9-11.

26 Cfr. G. Maspero, Dio trino perché vivo, Brescia 2018, 110-113. 
divine. ${ }^{27}$ Il Padre, il Figlio e lo Spirito sono così presentati in modo correlativo l'uno all'altro, in quanto ciascuno di essi si identifica con l'unica eterna ed infinita sostanza divina, distinguendosi dagli altri solo a livello di relazione.

Che si tratti di un'autentica novità ontologica può essere evidenziato confrontando tali affermazioni con il pensiero di Plotino e Porfirio secondo i quali l'essere generato implicava necessariamente inferiorità metafisica del generato rispetto al generante, insieme all'impossibilità che la relazione potesse essere elevata a livello sostanziale ${ }^{28} \mathrm{Il}$ primo principio doveva, per loro, essere assolutamente privo di relazioni. ${ }^{29} \mathrm{Il}$ punto qui in gioco è la distinzione tra ontologia e gnoseologia. Nella prospettiva patristica, infatti, l'essere non si identifica con l'intellegibile, ma è mistero di profondità infinita. La convertibilità degli universali è mantenuta in quanto il Logos è conoscenza ed espressione assoluta dell'Essere, ma come Persona divina, perché ora solo Dio può dire Dio. La conoscenza diventa relazione. E ciò vale anche per il mondo creato ed eminentemente per l'uomo creato ad immagine e somiglianza di Dio. Solo la relazione con il Dio uno e trino può rivelare la vera identità della creatura. Qui l'ontologia trinitaria appare in tutta la sua forza: la ricomprensione in chiave relazionale del primo principio induce, infatti, una rilettura del mondo a partire dal nuovo statuto ontologico della relazione stessa.

\section{VERIFICA MARIOLOGICA}

Il percorso fin qui tracciato, forse troppo ampio e ardito, ha preso le mosse dal ripensamento della metafisica classica operato dai Padri della Chiesa, i quali hanno così formulato un'ontologia relazionale caratterizzata dal gap, dall'apofatismo come suo riflesso a livello conoscitivo, e dall'inserimento della relazione in quanto coprincipio dell'essere insieme alla sostanza e inseparabilmente rispetto ad essa, fino al punto da identificarne l'immanenza. Si è dimostrato come tale ontologia relazionale costituisca una vera e propria ontologia trinitaria, in quanto non solo riformula l'ontologia del Dio uno e trino, ma pure rilegge il mondo alla luce di tale riformulazione.

Ma se questo è vero, tale nuova metafisica deve costituire un'ontologia mariana, perché, se lo sviluppo ontologico per pensare l'essere del Dio uno e trino alla luce della rivelazione ha condotto a ripesare il creato alla luce trinitaria, è naturale che in primo luogo ciò avvenga per la Madre di Dio.

27 Cfr. Gregorio di Nazianzo, Oratio 31 (De Spiritu Sancto), 2.

28 Cfr. Porfirio, Sententiae ad intelligibilia, 13.

29 Cfr. Porfirio, In Platonis Parmenidem commentaria, III, 32-IV, 4. 
Qui la versione relazionale dell'ontologia trinitaria, ora tratteggiata, si rivela particolarmente feconda. Anche storicamente, infatti, proprio il riconoscimento in forma piena del gap ontologico tra la Trinità e l'uomo è coinciso con le prime attribuzioni a Maria del suo essere immacolata, sempre vergine, tutta santa. ${ }^{30}$ Infatti, nel nuovo assetto ontologico, tali aggettivi non risultavano più equivoci perché rimaneva assolutamente chiaro che la fanciulla di Nazareth era creatura e che le sue perfezioni erano doni ricevuti nella sua sostanza mediante la singolarità e l'eccellenza della sua relazione con il Cristo.

Infatti, nel seno di Maria è entrato nel tempo l'Unigenito che nell'eternità è nel seno del Padre, secondo quanto si deduce dal Prologo giovanneo stesso. Qui la Madre di Dio può essere intravista nell'espressione che indica il venire ad abitare tra gli uomini del Logos che si fa carne, mediante il verbo che in greco richiama il "porre la tenda" (cfr. Gv 1,14), come durante l'esodo nel deserto l'arca e, quindi, la presenza di Dio, abitavano nell'accampamento ebreo. Si può riconoscere, così, una corrispondenza relazionale: il fatto che il Figlio è nel Padre e il Padre è nel Figlio si traduce nel Figlio che si fa carne in Maria la quale a sua volta, come creatura, è stata creata nel Figlio che si fa carne in Lei (cfr. Col 1,15-20).

In lei come luogo e come cuore, come frontiera, le processioni si prolungano nelle missioni. In lei, grazie al suo sì, diventa possibile la partecipazione alle relazioni divine. Nel suo affidarsi a Dio che la cerca per essere Madre del Verbo che si fa carne, la ricettività del Figlio il quale sempre si riceve e si restituisce al Padre fonda il valore ontologico della storia. La generazione eterna trova in Maria il suo libero prolungamento come generazione nel tempo. Il Padre e il Figlio sono sé stessi solo nella relazione di Amore assoluto che li unisce nell'immanenza dell'unica sostanza. E tale generazione è eterna in modo tale che, come visto, le relazioni sono sussistenti. Maria, invece, è creatura e quindi contingente. Ma quando Dio le chiede di essere Madre del Verbo che si fa carne, lei diventa Madre nello stesso momento e per la stessa ragione per la quale il Figlio di Dio diventa Figlio dell'Uomo. Così la filiazione di Gesù a Maria e la maternità di Maria nei confronti di Gesù sono relazioni che sorgono insieme, nel tempo, tra sostanze diverse. La novità ontologica interviene proprio qui, perché il Figlio di Dio che diventa Figlio di Maria è una cosa sola con il Padre, cioè è eterno. La sua Persona come relazione sussistente è immanente alla Trinità, in modo tale che da questa

30 Cfr. L.F. Mateo-Seco, La mariología de San Gregorio de Nisa, Scripta Theologica 10 (1978), 409-466; M. Gordillo, La virginidad transcendente de María Madre de Dios en S. Gregorio de Nisa y en la antigua tradición de la Iglesia, Estudios marianos 21 (1960), 117-155; G. Maspero, El Misterio de la Virgen Toda Limpia en Gregorio de Nisa, Scripta de Maria II,1 (2004), 183-205. 
relazione eterna si riversa nella relazione storica tra Maria e Gesù. La relazione tra la Madre e il Figlio è in un certo modo abbracciata dalla relazione tra il Padre e il Figlio, impregnandola di vita divina ed eterna. Da qui passa la salvezza cristiana. Chiaramente Maria e Gesù permangono come sostanze separate, ma la loro relazione è una volta per tutte unita alle relazione sussistenti intratrinitarie, in modo tale che l'unità e la vita di Dio sono comunicate agli uomini. Così tutto in Maria è relazione, fino al punto che, in un'unione senza confusione e una distinzione senza separazione, il suo essere creatura e figlia di Dio fiorisce nel diventare Madre di Dio, Madre della seconda Persona divina che è la Filiazione sussistente. Lei può essere, dunque, definita Madre della relazione e Regina delle relazioni.

È qui essenziale il gap e, quindi, la differenza tra immanenza ed economia, tra eternità e storia. Il Figlio nella generazione eterna riceve, infatti, assolutamente e perfettamente dal Padre l'infinita vita divina che proprio come Immagine perfetta del Padre stesso a Lui restituisce assolutamente e perfettamente. Qui non c'è perdita alcuna perché in Dio tutto è infinito e senza limite. Invece, come uomo il Cristo sempre riceve tutto sé stesso dal Padre e a Lui si restituisce, ma ora la vita umana è finita, in modo tale che il Mistero Pasquale rivela proprio l'identità trinitaria di Dio nella morte del Verbo che si è fatto carne. Tale radicale abbandono significa ed esprime in termini umani, quindi "al finito", la relazione di reciproco dono assoluto di sé del Padre e del Figlio, che nell'immanenza si dà "all'infinito". Maria, Madre del Verbo che è Dio, è al centro di questa traduzione dell'eterno dono mutuo delle Persone divine in termini umani. Infatti quella carne e quella vita umana hanno origine da lei, dal suo sì, dalla sua libertà, cioè dal suo dono totale e dal suo abbandono al Padre. Lei tutto riceve e tutto restituisce: sé stessa e suo Figlio, cioè la propria maternità. In questo è perfettamente figlia del Padre e perfettamente madre del Figlio, in quanto affida la relazione che definisce la sua identità, cioè la maternità, al Padre stesso, sottraendola in tal modo al potere della morte e del limite.

La tradizione ha espresso in forma intuitiva questa profondità ontologica di Maria chiamandola Figlia di Dio Padre, Madre di Dio Figlio e Sposa di Dio Spirito Santo. ${ }^{31}$ In lei tutto è relazione a ciascuna Persona divina, non per merito, ma per grazia. Il suo essere creato è spazio, carne, tempo, totalmente "trinitarizzati", ${ }^{2}$ cioè trasfigurati dalla terza Persona divina che ha coperto lei con la sua ombra riempiendola di grazia in pura trasparenza relazionale al Padre, al Figlio e allo Spi-

31 Francesco di Assisi, Scritti, 163. La formula era particolarmente amata anche dal già citato Josemaría Escrivá de Balaguer (si veda, ad esempio, Cammino, Milano 1956, § 496).

32 L'espressione è cara a Chiara Lubich ed ha una estrema profondità insieme mistica e teologica: cfr. P. Coda, A. Tapken, La Trinità e il pensare, Roma 1997, pp. 422-423. 
rito Santo stesso. Forse per questo l'espressione tradizionale potrebbe essere riformulata in Figlia di Dio Padre, Madre di Dio Figlio e Casa (Home) dello Spirito, dove l'ultimo termine va inteso in senso personale e famigliare. Proprio in tale Casa l'uomo contemporaneo, segnato dal postmoderno, può imparare il valore delle relazioni per perdere la paura delle differenze, contemplando colei che più di ogni altro ha avuto una relazione con Cristo personale e non istituzionale, secondo le aspirazioni della Riforma stessa. Il suo essere può venir letto, così, proprio come eminente luogo dell'ontologia trinitaria, come spazio personale, temporale e corporale, in relazione al quale è possibile la reale adorazione della Santissima Trinità.

\section{CONCLUSIONE}

Si è mostrato, dunque, che (a) i Padri della Chiesa non solo hanno lavorato sulla metafisica, ma addirittura l'hanno trasformata sviluppando una nuova ontologia per descrivere il Dio uno e trino e modificando la metafisica classica in modo tale da attribuire alla relazione un valore non meramente accidentale e che (b) a partire da ciò hanno riletto il creato, oggetto dell'indagine filosofica classica, in una luce nuova, evidenziando il ruolo del gap e dell'apofatismo, fino a (c) ricomprendere l'uomo stesso come rivela la loro riflessione su Maria, la Madre di Dio. Ciò permette di superare ogni approccio dialettico che opponga Scrittura e Metafisica, per poter essere una Chiesa, e una teologia, in uscita, che è capace di mostrare a chi ancora non crede la bellezza della novità cristiana. Proprio l'ontologia della relazione patristica può rivelarsi strumento prezioso per attrarre a Cristo in quest'epoca postmoderna, che nega tutte le differenze per paura che esse siano causa di conflitto. Invece i Padri della Chiesa e la Madre di Dio ci mostrano che proprio le differenze possono essere luoghi di incontro ed opportunità perché si sviluppino quelle relazioni vere che, in quanto tali, costituiscono un'autentica novità di essere e di vita.

\section{BIBLIOGRAFIA}

Aristotele, Categoriae, 1b 26-2a1 e 6a, 36-37.

Aristotele, Ethica Nicomachea, 1096a, 21-22.

Aristotele, Metaphysica, XII, 7: 1072a, 21-26.

Atanasio, Epistula ad Serapionem, II, 10,2; III, 1,3.

Benveniste E., Le vocabulaire des institutions indo-européennes, I, Paris 1969, pp. 210-211. Coda P., Per una lettura trinitaria del prologo di Giovanni, Milano 2007. 
Coda P., L'ontologia trinitaria: cos'è?, Sophia 4 (2012), 159-179.

Coda P., Tapken A., La Trinità e il pensare, Roma 1997.

Francesco, Evangelii gaudium.

Francesco di Assisi, Scritti, 163.

Escrivá de Balaguer J., Cammino, Milano 1956.

Giustino, Apologia, I, 46, 2,1-3,6.

Giustino, Dialogus cum Tryphone, 127.

Gnilka Ch., Chrêsis: die Methode der Kirchenväter im Umgang mit der antiken Kultur: Der Begriff des "rechten Gebrauchs”, Basel 2012.

Gordillo M., La virginidad transcendente de María Madre de Dios en S. Gregorio de Nisa y en la antigua tradición de la Iglesia, Estudios marianos 21 (1960), 117-155.

Gregorio di Nazianzo, Oratio 31 (De Spiritu Sancto), 2 i 9.

Gregorio di Nissa, Contra Eunomium, II, 1, 386, 9-11.

Gregorio di Nissa, Eunomio, in: Gregorio di Nissa, Contra Eunomium, I, 151,1-152, 1.

Harnack A. von, Lehrbuch der Dogmengeschichte, I, Freiburg i. B. 1888, pp. ix-x.

Maspero G., El Misterio de la Virgen Toda Limpia en Gregorio de Nisa, Scripta de Maria II, 1 (2004), 183-205.

Maspero G., Uno perché trino, Siena 2011.

Maspero G., Essere e relazione, Roma 2013.

Maspero G., Dio trino perché vivo, Brescia 2018.

Mateo-Seco L.F., La mariología de San Gregorio de Nisa, Scripta Theologica 10 (1978), 409-466.

Origene, Commentarii in evangelium Joannis, II, 10, 76, 2-7; XIII, 21, 140, 1-12.

Pazzini D., In principio era il Logos: Origene e il prologo del Vangelo di Giovanni, Brescia 1983.

Pazzini D., L'interpretazione del Prologo di Giovanni in Origene e nella patristica greca, Annali di storia dell'esegesi 11 (1994), 45-56.

Platone, Timaeus, $90 \mathrm{a}-\mathrm{b}$.

Porfirio, In Platonis Parmenidem commentaria, III, 32-IV, 4.

Porfirio, Sententiae ad intelligibilia, 13.

Ramelli I., Origen's anti-Subordinationism and its Heritage in the nicene and Cappadocian Line, Vigiliae christianae 65 (2011), 21-49.

Renczes G.Ph., La patristica e la metafisica nel secolo XX, Gregorianum 90 (2009), 76-85.

Simonetti M., voce "Spirito Santo", in: Dizionario Origene, ed. A. Monaci Castagno, Roma 2000, pp. 450-456. 
Parole chiavi: Padri della Chiesa, metafisica, esegesi della Sacra Scrittura

\section{CHURCH FATHERS AND METAPHYSICS: UNAVOIDABLE RELATIONSHIP}

\section{Summary}

Reflections which concern using metaphysics in the teaching of the Church Fathers are highly important, because this relationship is deeply rooted in their exegesis. The relationship was negated specifically by the Reformation, which insisted that biblical language was allegedly replaced by pagan Greek philosophy. In fact, the Church Fathers were not only working on metaphysics itself but also they modified its classical form, producing some kind of new ontology, which is helpful in more precise theological depiction of Trinitarian God. On that basis they had initiated a new interpretation of metaphysics, showing a new perspective in looking at theological issues, such as creation, the mystery of human being, or Mariology.

Keywords: Church Fathers, metaphysics, exegesis

\section{OJCOWIE KOŚCIOŁA I METAFIZYKA: RELACJA NIEUNIKNIONA}

\section{Streszczenie}

Refleksje dotyczące zastosowania przez ojców Kościoła metafizyki w ich nauczaniu są bardzo istotne, ponieważ związek ten jest głęboko zakorzeniony w ich egzegezie. Została ona zakwestionowana szczególnie w obszarze reformacji, która utrzymywała, jakoby język biblijny został zastąpiony przez grecką filozofię pogańską. Ojcowie Kościoła nie tylko pracowali nad samą metafizyką, ale ponadto zmodyfikowali jej klasyczną formę, opracowując niejako nową ontologię, pomocną w bardziej precyzyjnym, teologicznym opisie Trójjedynego Boga. Na tej podstawie dokonali pogłębionej interpretacji, ukazując w nowym świetle wiele kwestii teologicznych (np. dzieło stworzenia, tajemnica człowieka, mariologia).

Słowa kluczowe: ojcowie Kościoła, metafizyka, egzegeza Pisma Świętego 